\title{
Meson Spectroscopy from Lattice QCD
}

Received: 8 December 2016 / Accepted: 7 March 2017 / Published online: 24 March 2017

(C) The Author(s) 2017. This article is an open access publication

\begin{abstract}
Some recent progress in using lattice QCD to perform first-principles calculations of the spectra of mesons is discussed. In particular, I highlight some new results on resonances, near-threshold states and related scattering phenomena - this is a theoretically and experimentally interesting area where we have made significant advances in the last few years.
\end{abstract}

\section{Introduction}

There has been a resurgence of interest in hadron spectroscopy in the last decade or so driven by the emergence of a wealth of high-quality experimental data. In particular, a number of unexpected states have been observed, e.g. various charmonium and bottomonium-like structures (" $X, Y, Z$ 's") and the charm-strange $D_{s 0}(2317)[49,53]$, and there are also longer-standing puzzles such as the makeup of the light scalar mesons [55,57]. There has been a lot of theoretical work and speculation about their nature $[6,50]$ - possibilities include tetraquarks (containing two quarks and two antiquarks), molecular states of hadrons, hadro-quarkonia and hybrid mesons (where the gluonic field is excited). States with exotic quantum numbers, i.e. those which cannot arise from solely a quark-antiquark pair, are particularly interesting because they are a smoking gun for physics beyond a model of a quark and an antiquark moving in a potential. For example, exotic spin $(J)$, parity $(P)$, chargeconjugation $(C)$ combinations (e.g. $J^{P C}=0^{--}, 0^{+-}, 1^{-+}, 2^{+-}$) or exotic flavour states (such as charged charmonium and bottomonium-like states).

Lattice Quantum Chromodynamics (Lattice QCD) is a method which enables first-principles computations of the spectra and properties of hadrons: four-dimensional space-time is discretised on a finite four-dimensional hypercubic lattice and the calculation of quantities in the path integral formulation then becomes an ordinary (but very large) integration problem. If a Euclidean (imaginary-time) space-time metric is used, the integrals can be evaluated effectively using importance-sampling Monte Carlo methods. The masses and other properties of hadrons then follow from analyzing correlation functions involving interpolating operators built from quark and gluon fields. Precise calculations of the spectrum of low-lying hadrons have long been benchmarks of lattice QCD (e.g. see Refs. [17,23]) but only relatively recently has there been significant progress in using lattice QCD to study excited hadrons.

Most hadrons are unstable with respect to the strong interaction and appear as resonances in the scattering of two or more lighter hadrons. In particular, many of the recently-observed puzzling states appear close to or above the threshold for strong decay - this must be taken into account in theoretical approaches and when analyzing experimental data. In recent years there have been significant advances in using lattice QCD to study resonances, near-threshold states and related scattering phenomena and I will discuss some highlights of this

This article belongs to the Topical Collection "Light Cone 2016".

C. E. Thomas $(\varangle)$

DAMTP, University of Cambridge, Wilberforce Road, Cambridge CB3 0WA, UK

E-mail: c.e.thomas@damtp.cam.ac.uk 
work in these proceedings. These calculations often use unphysically-heavy light quarks, corresponding to a pion mass, $m_{\pi}$, larger than the experiment pion mass and do not usually have a precise control over all the systematic uncertainties - details will not be given here and I refer to the references for further information.

After a brief description of some of the lattice QCD methodology in Sect. 2, in Sect. 3 I discuss calculations of the $\rho$ resonance in $\pi \pi$ scattering. Some other channels relevant for light and strange mesons are discussed in Sect. 4 followed by heavy-light and charmonium-like resonances in Sect. 5. I conclude in Sect. 6 with an outlook. ${ }^{1}$

\section{Excited Spectroscopy and Scattering in Lattice QCD}

In lattice QCD the discrete spectrum of states in a finite volume follows from analyzing the dependence on the time separation, $t$, of two-point correlation functions,

$$
C_{i j}(t)=\left\langle 0\left|\mathcal{O}_{i}(t) \mathcal{O}_{j}^{\dagger}(0)\right| 0\right\rangle .
$$

Here the interpolating operator $\mathcal{O}_{j}^{\dagger}(0)$ creates the states of interest at time 0 and $\mathcal{O}_{i}(t)$ annihilates them at time $t$-all states with the quantum numbers of the operators contribute to the correlation function. The variational method $[5,43,44]$ enables excited states to be extracted reliably: a matrix of correlation functions involving a basis of $N$ operators with the required quantum numbers is computed, $C_{i j}(t)$, where $i, j=1,2, \ldots, N$. A generalised eigenvalue problem,

$$
C_{i j}(t) v_{j}^{(n)}=\lambda^{(n)}(t) C_{i j}\left(t_{0}\right) v_{j}^{(n)},
$$

is then solved for an appropriate reference time $t_{0}$. The time dependence of an eigenvalue, $\lambda^{(n)}(t)$, is related to the energy of the $n$ 'th state. The eigenvectors, $v_{i}^{(n)}$, are related to the operator-state overlaps, $Z_{i}^{(n)} \equiv\left\langle 0\left|\mathcal{O}_{i}\right| n\right\rangle$, which can be used to probe the structure of states and also give the optimal (in a variational sense) linear combination of operators to create the $n$ 'th state. To accurately determine the spectrum, the basis of operators must have a sufficiently wide range of structures to disentangle the various states that appear.

Although it is not possible to compute scattering properties directly in the Euclidean formulation of lattice QCD, the Lüscher method [40-42] and its extensions [7,8,16,25,27,32,38,39,62] allow indirect access to infinite-volume scattering amplitudes from finite-volume spectra, at least in the case of any number of twohadron coupled channels. For elastic scattering, there is a one-to-one correspondence ${ }^{2}$ between an energy level with centre-of-mass energy $E_{\mathrm{cm}}$ and the scattering phase shift $\delta$ at $E_{\mathrm{cm}}$ or equivalently the scattering $t$-matrix, $t\left(E_{\mathrm{cm}}\right)$. For coupled-channel two-hadron scattering, extracting an energy level $E_{\mathrm{cm}}$ constrains the scattering $t$-matrix at $E_{\mathrm{cm}}$ but this is in general an under-constrained problem. For example, with two coupled channels there are three energy-dependent parameters but only one constraint at each $E_{\mathrm{cm}}$. One solution is to parameterize the $E_{\mathrm{cm}}$-dependence of the $t$-matrix with a relatively small number of parameters-the spectrum resulting from this $t$-matrix is fit to the computed spectrum by varying these parameters. With the $t$-matrix in hand, its singularity structure can be investigated to determine the bound state and resonant content. The former corresponds to a pole below threshold on the real axis of the physical Riemann sheet, whereas the latter corresponds to poles away from the real axis on unphysical sheets.

\section{The $\rho$ Resonance in $\pi \pi$ Scattering}

One of the simplest resonances, and the one that has attracted the most initial attention from lattice QCD [1, $2,24,33,56]$, is the $\rho(770)$ which appears in $\pi \pi$ scattering in $L=1$ ( $P$-wave) with $J^{P C}=1^{--}$and isospin $I=1$; empirically the $\rho$ decays almost entirely to $\pi \pi$ [53]. The results of a recent lattice QCD calculation [65] of the elastic $I=1 \pi \pi$ scattering phase shift by the Hadron Spectrum Collaboration (HSC) are shown in Fig. 1. The computations have dynamical strange and degenerate up and down quarks, " $N_{f}=2+1$ ", corresponding to $m_{\pi} \approx 240 \mathrm{MeV}$, and used 469 gauge field configurations; the spatial (temporal) lattice extent is $L \sim 4 \mathrm{fm}$ $(T \sim 9 \mathrm{fm})$. The plot also shows results from an earlier calculation with $m_{\pi} \approx 390 \mathrm{MeV}$. A combination of

\footnotetext{
1 This talk was based on a similar talk I gave at the Confinement XII conference and this write-up is similar to Ref. [63].

2 Ignoring complications arising from the mixing of partial waves due to the reduced symmetry of a finite box which we will not discuss here.
} 


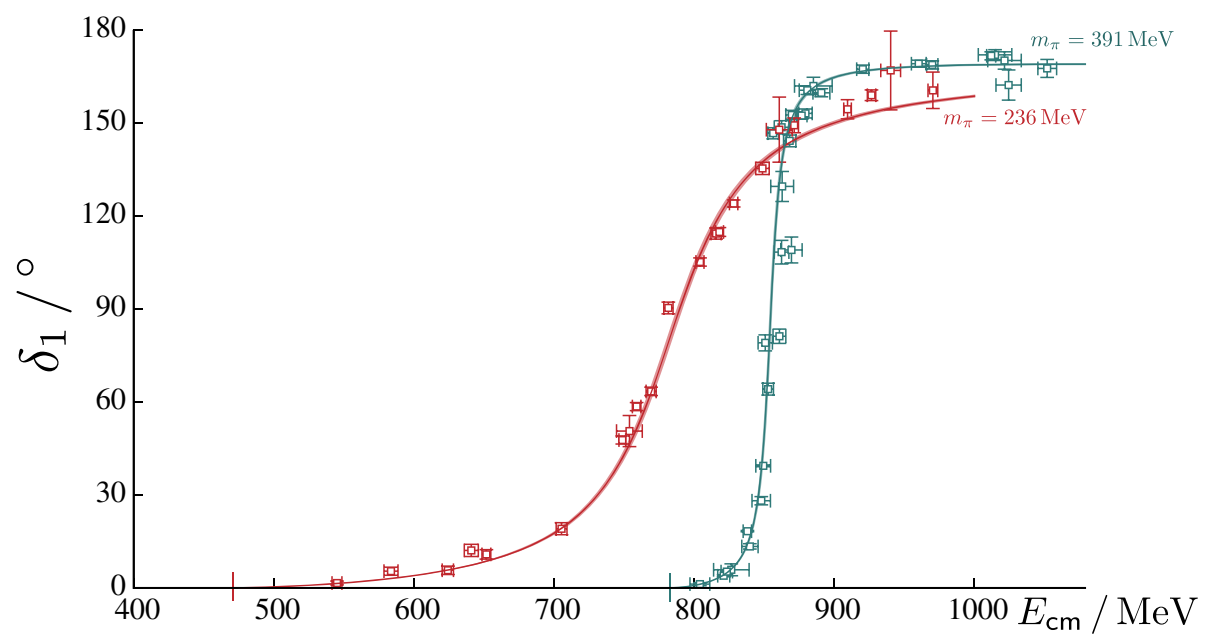

Fig. 1 From Ref. [65]. The $P$-wave $I=1 \pi \pi$ elastic scattering phase shift plotted from $\pi \pi$ threshold to the inelastic $K \bar{K}$ threshold, from dynamical lattice QCD calculations with $m_{\pi} \approx 240 \mathrm{MeV}$ [65] and $m_{\pi} \approx 390 \mathrm{MeV}$ [20]. Points are from an energy-level by energy-level analysis and the curves are from a relativistic Breit Wigner parameterization as described in [65]

techniques were used to calculate extensive finite-volume spectra with high statistical precision: the distillation approach [54] was used to compute correlation matrices involving large bases of carefully-constructed fermionbilinear [18,64] and multi-hadron interpolating operators [19,20] (between 10 and 37 operators in each of 12 different irreducible representations of the relevant symmetry group). The correlation matrices were analysed using the variational method and the resulting spectra, with 22 energy levels in the elastic region, enabled the energy dependence of the scattering phase shift to be mapped out in detail via the the Lüscher method.

The figure shows convincingly the rapid rise in the phase shift from $0^{\circ}$ through $90^{\circ}$ to $180^{\circ}$ that is expected in the presence of a single isolated resonance. The resonance mass $\left(M_{R}=790 \pm 2 \mathrm{MeV}\right)$ is not too different from experiment $(775.49 \pm 0.3 \mathrm{MeV})$ but the width is significantly smaller $(\Gamma=87 \pm 2 \mathrm{MeV}$ compared to $149.1 \pm 0.8 \mathrm{MeV}$ experimentally). This is simply because the pion mass in this study is larger than the physical value and so the phase space for decay is reduced. The coupling, $g$, with the phase space factor divided out, defined by $\Gamma=\frac{g^{2}}{6 \pi} \frac{p_{\mathrm{cm}}^{3}}{M_{R}}$, where $p_{\mathrm{cm}}$ is the cm-frame scattering momentum, shows little dependence on $m_{\pi}$ and is in reasonable agreement with experiment.

Ref. [65] also extends the analysis to the coupled-channel $\pi \pi, K \bar{K}$ energy region using 34 energy levels. Negligible coupling between the channels is found in the energy range explored.

The RQCD Collaboration have recently studied the $\rho$ resonance with $m_{\pi} \approx 150 \mathrm{MeV}$, close to the physical value [3]. However, in these " $N_{f}=2$ " computations only the light up and down quarks were dynamical whereas the strange quarks were quenched (not present in the sea). As can be seen from the comparison of different lattice QCD results in Ref. [3], the resonance mass is sensitive to whether or not the strange quarks are quenched (unlike $g$ which appears to be insensitive to this and to $m_{\pi}$ ). The same effect was observed in recent $N_{f}=2$ computations by Guo et. al. [26] with $m_{\pi}=226$ and $315 \mathrm{MeV}$, as is discussed in Ref. [31].

Going beyond the resonance mass and width, recently the form factor of an unstable hadron has been calculated for the first time in lattice QCD [10,12]. From computations of the $\pi \pi \rightarrow \pi \gamma^{*}$ transition amplitude, the resonant $\rho \rightarrow \pi \gamma^{*}$ transition form factor and the radiative decay width were extracted.

\section{Other Scattering Channels Relevant for Light and Strange Mesons}

Moving to other scattering channels relevant for light and strange mesons, using a similar setup to that described above with $m_{\pi} \approx 390 \mathrm{MeV}$ and three lattice volumes, the HSC investigated coupled-channel $K \pi, K \eta$ scattering with $I=1 / 2[21,66]$, the first study of coupled-channel scattering on the lattice. In total 73 energy levels were extracted and these enabled the energy dependence of the scattering matrix to be mapped out. A number of interesting features emerged including a broad $J^{P}=0^{+}$scalar resonance in $S$-wave [c.f. the $K_{0}^{*}(1430)$ ], a $P$-wave $1^{-}$vector bound state [c.f. the $K^{*}(892)$ ], a narrow $2^{+}$tensor resonance in $D$-wave [c.f. the $K_{2}^{*}(1430)$ ] and a suggestion that the $\kappa$, a scalar resonance with physical-mass light quarks, corresponds to a virtual bound 

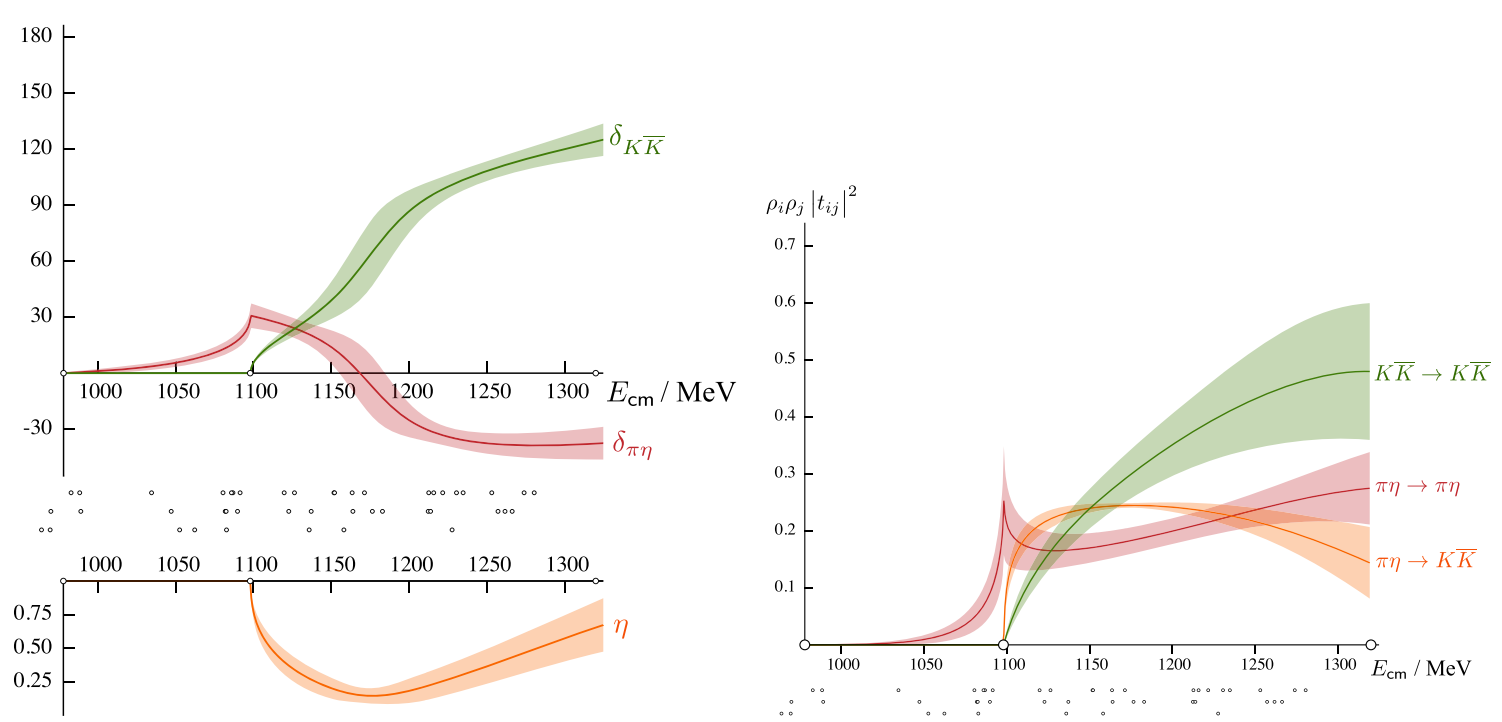

Fig. 2 From Ref. [22]. The $S$-wave $I=1 \pi \eta, K \bar{K}$ coupled-channel scattering amplitudes $\left(J^{P C}=0^{++}\right)$from a lattice calculation with $m_{\pi} \approx 390 \mathrm{MeV}$. Left panel shows phase shifts, $\delta_{i}$, and inelasticity, $\eta$, and the right panel shows quantities proportional to cross sections. Open circles on the horizontal axis indicate $\pi \eta, K \bar{K}$ and $\pi \eta^{\prime}$ thresholds. The dots below the figures show energy levels used to constrain the amplitudes

state $^{3}$ when $m_{\pi} \approx 390 \mathrm{MeV}$. The latter observation is consistent with how the $\kappa$ behaves in unitarised chiral perturbation theory as the $\pi$ mass is varied [48]. The exotic-flavour $I=3 / 2 K \pi$ channel was also studied.

The RQCD Collaboration have studied $P$-wave elastic $K \pi$ scattering in a $N_{f}=2$ calculation with light quarks corresponding to $m_{\pi} \approx 150 \mathrm{MeV}$ [3]. They find a vector resonance, consistent with the expectation that the bound state found by the HSC with $m_{\pi} \approx 390 \mathrm{MeV}$ becomes a resonance as the light-quark masses are reduced.

The HSC have also studied coupled $\pi \eta, K \bar{K}, \pi \eta^{\prime}$ scattering in $I=1$ with $m_{\pi} \approx 390 \mathrm{MeV}$ on three lattice volumes: $L \approx 1.9,2.4$ and $2.9 \mathrm{fm}$ using respectively 479,603 and 553 configurations [22]. The $S$-wave coupled-channel $\pi \eta, K \bar{K}$ scattering amplitudes constrained by 47 energy levels are shown in Fig. 2 . Here the phase shifts, $\delta_{i}$, and inelasticity, $\eta$, are defined in terms of elements of the $t$-matrix, ${ }^{4}$

$$
t_{i j}= \begin{cases}\frac{\eta e^{2 i \delta_{i}}-1}{2 i \rho_{i}} & (i=j) \\ \frac{\sqrt{1-\eta^{2}} e^{i\left(\delta_{i}+\delta_{j}\right)}}{2 \sqrt{\rho_{i} \rho_{j}}} & (i \neq j)\end{cases}
$$

where $i, j$ label scattering channels and $\rho_{i}=2 p_{\mathrm{cm}, i} / E_{\mathrm{cm}}$ is the phase space factor for channel $i$. The amplitudes show a cusp-like enhancement in $\pi \eta \rightarrow \pi \eta$ near $K \bar{K}$ threshold and a rapid turn on of amplitudes to $K \bar{K}$. This behaviour originates from an $a_{0}(980)$-like resonance which is strongly coupled to both $\pi \eta$ and $K \bar{K}$-the first strongly-coupled meson-meson scattering system extracted in a lattice QCD calculation. The corresponding pole appears on a single unphysical Riemann sheet, unlike a canonical two-channel resonance where poles would be expected on two unphysical sheets, and this may be a sign that the state binds through the longrange interaction between a pair of mesons. Ref. [22] also presents results with the $\pi \eta^{\prime}$ channel included and considers $D$-wave scattering where a narrow tensor resonance is found.

Using a similar setup again, the HSC have recently determined the elastic $\pi \pi S$-wave $I=0$ scattering phase shift for the first time in a lattice QCD computation [11]. Two different light quark masses were used corresponding to $m_{\pi} \approx 240 \mathrm{MeV}$ and $390 \mathrm{MeV}$. For the lighter mass the scattering amplitude has a pole on the unphysical Riemann sheet with a large imaginary part, corresponding to a broad resonance, in qualitative agreement with experiment. At the heavier mass a pole is found below threshold on the real axis of the physical sheet, corresponding to a bound state. Work is ongoing to extend this calculation into the coupled-channel region above $K \bar{K}$ threshold where a $f_{0}(980)$-like resonance is expected, the final component of a study of the scalar meson nonet using lattice QCD.

\footnotetext{
3 A pole below threshold on the real axis of an unphysical Riemann sheet.

${ }^{4}$ Note that $\eta=1$ would indicate no coupling between channels.
} 


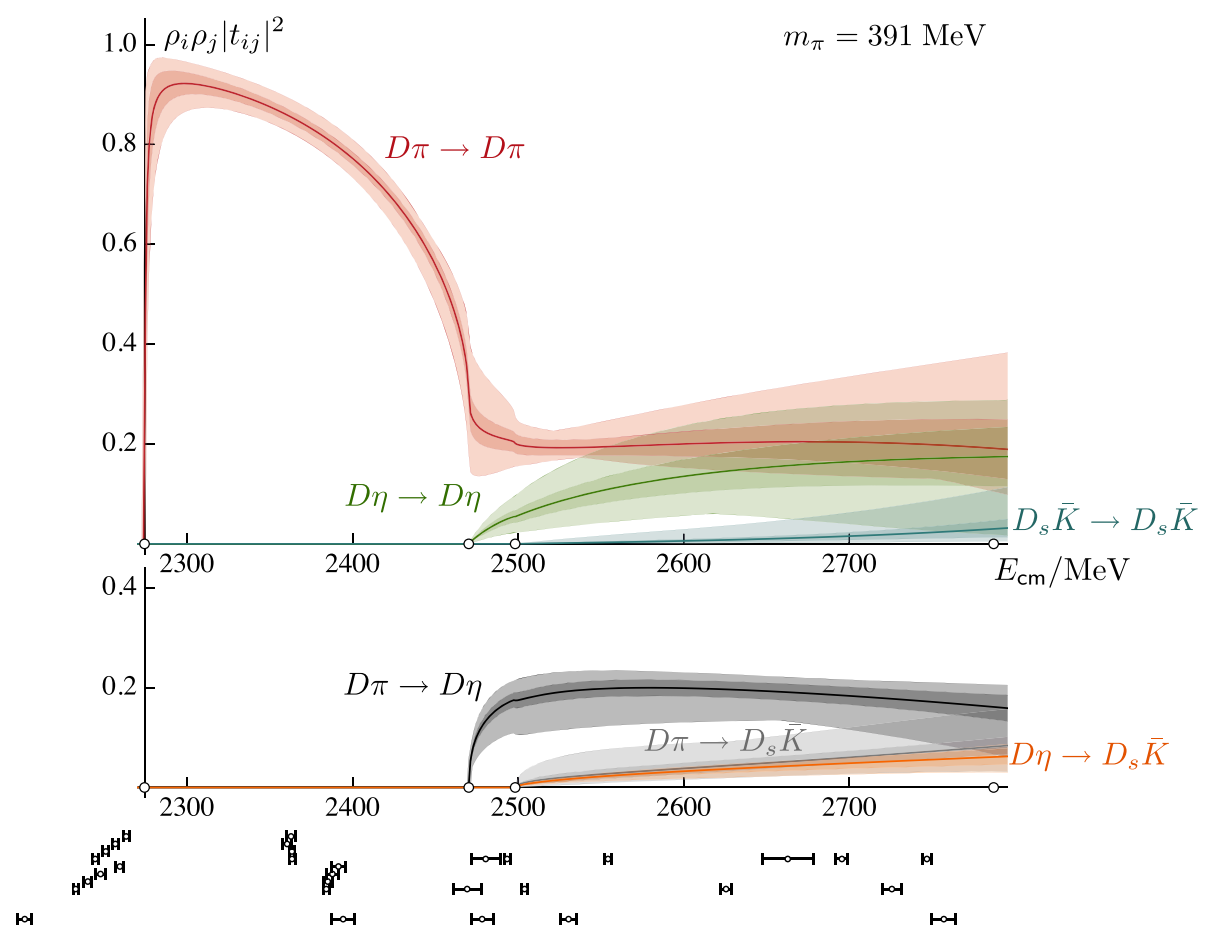

Fig. 3 From Ref. [47]. The $S$-wave $I=1 / 2 D \pi, D \eta, D_{s} \bar{K}$ coupled-channel scattering amplitudes from a lattice calculation with $m_{\pi} \approx 390 \mathrm{MeV}$. Quantities proportional to the diagonal (off-diagonal) cross sections are plotted in the top (bottom) panel. Open circles on the horizontal axes indicate $D \pi, D \eta, D_{s} \bar{K}$ and $D^{*} \pi \pi$ thresholds. The black points below the plot show energy levels used to constrain the amplitudes

\section{Heavy-Light and Charmonium-Like Resonances}

Turning to channels relevant for heavy-light and quarkonium mesons, we begin with the charm-light $(D)$ and charm-strange $\left(D_{s}\right)$ sectors. The $D_{s 0}^{*}(2317)$ and $D_{s 1}(2460)$ present a long standing-puzzle: they were expected to be broad resonances, like the analogous $D_{0}^{*}(2400)$ and $D_{1}$ (2430) mesons, but were observed to be narrow states below the respective $D K$ and $D^{*} K$ thresholds [53]. Some early lattice calculations of elastic $D^{*} \pi$ and $D^{*} K$ scattering can be found in Refs. [34,45,46].

The HSC have recently investigated $I=1 / 2$ coupled-channel $D \pi, D \eta, D_{s} \bar{K}$ scattering with light-quark masses corresponding to $m_{\pi} \approx 390 \mathrm{MeV}$ on three lattice volumes: $L \approx 1.9,2.4$ and $2.9 \mathrm{fm}$ using respectively 479, 603 and 553 configurations [47]. Figure 3 shows the $S$-wave scattering amplitudes constrained by 47 energy levels - the broad feature in the $D \pi \rightarrow D \pi$ amplitude is associated with a $J^{P}=0^{+}$bound state very close to $D \pi$ threshold. Although, by contrast, the associated experimental state, $D_{0}^{*}(2400)$, is a resonance, they are similar in that they influence a broad energy range and couple predominantly to $D \pi$. The pole position lies between what is observed experimentally for the $D_{s 0}^{*}(2317)$ and the $D_{0}^{*}(2400)$. In $P$-wave $\left(J^{P}=1^{-}\right)$, a deeply bound state was found and can be compared to the experimentally observed narrow resonance, $D^{*}(2007)$. A narrow $2^{+}$resonance was found in $D$-wave. The $I=3 / 2$ elastic $D \pi$ channel was also investigated and a weakly repulsive interaction found in $S$-wave.

In the charmonium sector there have been a number of lattice QCD investigations [13-15,35,51,52,5961] and there have been a couple in the bottom sector [36,37]. However, the calculations are somewhat less advanced than in the other sectors and studies have generally been exploratory, inconclusive or have not determined the phenomena in detail or robustly, and I will not discuss these here. More robust and detailed computations are required-one challenge is the number of channels to which a resonance can potentially couple and all these channels need to be considered in the calculations.

\section{Conclusions}

I have highlighted some recent lattice QCD studies of resonances and related scattering phenomena, an area which has seen significant advances in the last few years and where prospects for further progress are very 
good. Although it may be seen as a disadvantage that many of the computations used unphysically-heavy light quarks, corresponding to $m_{\pi}$ larger than the experimental value, and so cannot be compared quantitatively to experiment, it turns out that studying how states evolve as $m_{\pi}$ varies is a useful tool in discerning their nature. As we go further up in the spectrum and undertake calculations with $m_{\pi}$ closer to the physical value, in general calculations become more difficult because additional channels become relevant and, in particular, channels involving more than two hadrons open up. Work on the formalism for such situations is ongoing $[4,9,28-30,58]$.

To stringently probe the structure of states we must go beyond solely computing masses and consider other properties of hadrons such as form factors and related quantities. There are also good prospects here with the recent appearance of the first lattice QCD calculation of a transition form factor involving a resonance [10,12].

Acknowledgements I thank the organisers for the invitation to give a talk at this meeting and my colleagues in the Hadron Spectrum Collaboration. I acknowledge support from the UK Science and Technology Facilities Council (STFC) [Grant ST/L000385/1].

Open Access This article is distributed under the terms of the Creative Commons Attribution 4.0 International License (http:// creativecommons.org/licenses/by/4.0/), which permits unrestricted use, distribution, and reproduction in any medium, provided you give appropriate credit to the original author(s) and the source, provide a link to the Creative Commons license, and indicate if changes were made.

\section{References}

1. S. Aoki et al., Lattice QCD calculation of the $\rho$ meson decay width. Phys. Rev. D 76(094), 506 (2007). doi:10.1103/ PhysRevD.76.094506. arXiv:0708.3705

2. S. Aoki et al., $\rho$ meson decay in $2+1$ flavor lattice QCD. Phys. Rev. D84(094), 505 (2011). doi:10.1103/PhysRevD.84. 094505. arXiv: 1106.5365

3. G.S. Bali, S. Collins, A. Cox, G. Donald, M. Gckeler, C.B. Lang, A. Schfer, $\rho$ and $K^{*}$ resonances on the lattice at nearly physical quark masses and $N_{f}=2$. Phys. Rev. D 93(5), 054509 (2016). doi:10.1103/PhysRevD.93.054509. arXiv:1512.08678

4. S.R. Beane, W. Detmold, M.J. Savage, n-boson energies at finite volume and three-boson interactions. Phys. Rev. D 76(074), 507 (2007). doi:10.1103/PhysRevD.76.074507. arXiv:0707.1670

5. B. Blossier, M. Della Morte, G. von Hippel, T. Mendes, R. Sommer, On the generalized eigenvalue method for energies and matrix elements in lattice field theory. JHEP 04, 094 (2009). doi:10.1088/1126-6708/2009/04/094. arXiv:0902.1265

6. N. Brambilla et al., QCD and strongly coupled gauge theories: challenges and perspectives. Eur. Phys. J. C74(10), 2981 (2014). doi:10.1140/epjc/s10052-014-2981-5. arXiv:1404.3723

7. R.A. Briceno, Two-particle multichannel systems in a finite volume with arbitrary spin. Phys. Rev. D 89(7), 074507 (2014). doi:10.1103/PhysRevD.89.074507. arXiv:1401.3312

8. R.A. Briceno, Z. Davoudi, Moving multichannel systems in a finite volume with application to proton-proton fusion. Phys. Rev. D 88(9), 094507 (2013a). doi:10.1103/PhysRevD.88.094507. arXiv:1204.1110

9. R.A. Briceno, Z. Davoudi, Three-particle scattering amplitudes from a finite volume formalism. Phys. Rev. D 87(9), 094507 (2013b). doi:10.1103/PhysRevD.87.094507. arXiv:1212.3398

10. R.A. Briceno, J.J. Dudek, R.G. Edwards, C.J. Shultz, C.E. Thomas, D.J. Wilson, The resonant $\pi^{+} \gamma \rightarrow \pi^{+} \pi^{0}$ amplitude from quantum chromodynamics. Phys. Rev. Lett. 115(242), 001 (2015). doi:10.1103/PhysRevLett.115.242001. arXiv:1507.06622

11. R.A. Briceno, J.J. Dudek, R.G. Edwards, D.J. Wilson, Isoscalar $\pi \pi$ scattering and the $\sigma$ meson resonance from QCD. Phys. Rev. Lett. 118(2), 022002 (2017). doi:10.1103/PhysRevLett.118.022002

12. R.A. Briceo, J.J. Dudek, R.G. Edwards, C.J. Shultz, C.E. Thomas, D.J. Wilson, The $\pi \pi \rightarrow \pi \gamma^{\star}$ amplitude and the resonant $\rho \rightarrow \pi \gamma^{\star}$ transition from lattice QCD. Phys. Rev. D 93(11), 114508 (2016). doi:10.1103/PhysRevD.93.114508. arXiv: 1604.03530

13. T. Chen et al., A lattice study of $\left(\bar{D}_{1} D^{*}\right)^{ \pm}$near-threshold scattering. Phys. Rev. D 93(11), 114501 (2016). doi:10.1103/ PhysRevD.93.114501. arXiv:1602.00200

14. Y. Chen et al., Low-energy scattering of the $\left(D \bar{D}^{*}\right)^{ \pm}$system and the resonance-like structure $Z_{c}(3900)$. Phys. Rev. D 89(9), 094506 (2014). doi:10.1103/PhysRevD.89.094506. arXiv:1403.1318

15. Y. Chen et al., Low-energy scattering of $\left(D^{*} \bar{D}^{*}\right)^{ \pm}$system and the resonance-like structure $Z_{c}(4025)$. Phys. Rev. D 92(5), 054507 (2015). doi:10.1103/PhysRevD.92.054507. arXiv:1503.02371

16. N.H. Christ, C. Kim, T. Yamazaki, Finite volume corrections to the two-particle decay of states with non-zero momentum. Phys. Rev. D72(114), 506 (2005). doi:10.1103/PhysRevD.72.114506. arXiv:hep-lat/0507009

17. R. Dowdall, C. Davies, T. Hammant, R. Horgan, Precise heavy-light meson masses and hyperfine splittings from lattice QCD including charm quarks in the sea. Phys. Rev. D86(094), 510 (2012). doi:10.1103/PhysRevD.86.094510. arXiv:1207.5149

18. J.J. Dudek, R.G. Edwards, M.J. Peardon, D.G. Richards, C.E. Thomas, Toward the excited meson spectrum of dynamical QCD. Phys. Rev. D 82, 034508 (2010). doi:10.1103/PhysRevD.82.034508. arXiv:1004.4930

19. J.J. Dudek, R.G. Edwards, C.E. Thomas, $S$ and D-wave phase shifts in isospin-2 pi pi scattering from lattice QCD. Phys. Rev. D 86(034), 031 (2012). doi:10.1103/PhysRevD.86.034031. arXiv:1203.6041

20. J.J. Dudek, R.G. Edwards, C.E. Thomas, Energy dependence of the $\rho$ resonance in $\pi \pi$ elastic scattering from lattice QCD. Phys. Rev. D 87(3), 034505, (2013). doi:10.1103/PhysRevD.87.034505, doi:10.1103/PhysRevD.90.099902, [Erratum: Phys. Rev.D90,no.9,099902(2014)], arXiv:1212.0830

21. J.J. Dudek, R.G. Edwards, C.E. Thomas, D.J. Wilson, Resonances in coupled $\pi K-\eta K$ scattering from quantum chromodynamics. Phys. Rev. Lett. 113(18), 182001 (2014). doi:10.1103/PhysRevLett.113.182001. arXiv:1406.4158 
22. J.J. Dudek, R.G. Edwards, D.J. Wilson, An $a_{0}$ resonance in strongly coupled $\pi \eta, K \bar{K}$ scattering from lattice QCD. Phys. Rev. D 93(9), 094506 (2016). doi:10.1103/PhysRevD.93.094506. arXiv:1602.05122

23. S. Durr et al., Ab-initio determination of light hadron masses. Science 322, 1224-1227 (2008). doi:10.1126/science.1163233. arXiv:0906.3599

24. X. Feng, K. Jansen, D.B. Renner, Resonance parameters of the rho-meson from lattice QCD. Phys. Rev. D 83, 094505 (2011). doi:10.1103/PhysRevD.83.094505. arXiv:1011.5288

25. Z. Fu, Rummukainen-Gottlieb's formula on two-particle system with different mass. Phys. Rev. D 85(014), 506 (2012). doi:10.1103/PhysRevD.85.014506. arXiv:1110.0319

26. D. Guo, A. Alexandru, R. Molina, M. Dring, Rho resonance parameters from lattice QCD. Phys. Rev. D 94(3), 034501 (2016). doi:10.1103/PhysRevD.94.034501. arXiv:1605.03993

27. M.T. Hansen, S.R. Sharpe, Multiple-channel generalization of Lellouch-Luscher formula. Phys. Rev. D 86(016), 007 (2012). doi:10.1103/PhysRevD.86.016007. arXiv:1204.0826

28. M.T. Hansen, S.R. Sharpe, Relativistic, model-independent, three-particle quantization condition. Phys. Rev. D 90(11), 116003 (2014). doi:10.1103/PhysRevD.90.116003. arXiv:1408.5933

29. M.T. Hansen, S.R. Sharpe, Expressing the three-particle finite-volume spectrum in terms of the three-to-three scattering amplitude. Phys. Rev. D 92(11), 114509 (2015). doi:10.1103/PhysRevD.92.114509. arXiv:1504.04248

30. M.T. Hansen, S.R. Sharpe, Perturbative results for two and three particle threshold energies in finite volume. Phys. Rev. D 93(014), 506 (2016). doi:10.1103/PhysRevD.93.014506. arXiv:1509.07929

31. B. Hu, R. Molina, M. Dring, A. Alexandru, Two-flavor simulations of the $\rho(770)$ and the role of the $K \bar{K}$ channel. Phys. Rev. Lett. 117(12), 122001 (2016). doi:10.1103/PhysRevLett.117.122001. arXiv:1605.04823

32. C. Kim, C. Sachrajda, S.R. Sharpe, Finite-volume effects for two-hadron states in moving frames. Nucl. Phys. B727, 218-243 (2005). doi:10.1016/j.nuclphysb.2005.08.029. arXiv:hep-lat/0507006

33. C. Lang, D. Mohler, S. Prelovsek, M. Vidmar, Coupled channel analysis of the rho meson decay in lattice QCD. Phys. Rev. D84(054), 503 (2011). doi:10.1103/PhysRevD.84.054503. arXiv:1105.5636

34. C.B. Lang, L. Leskovec, D. Mohler, S. Prelovsek, R.M. Woloshyn, Ds mesons with DK and D*K scattering near threshold. Phys. Rev. D 90(3), 034510 (2014). doi:10.1103/PhysRevD.90.034510. arXiv:1403.8103

35. C.B. Lang, L. Leskovec, D. Mohler, S. Prelovsek, Vector and scalar charmonium resonances with lattice QCD. JHEP 09, 089 (2015). doi:10.1007/JHEP09(2015)089. arXiv:1503.05363

36. C.B. Lang, D. Mohler, S. Prelovsek, R.M. Woloshyn, Predicting positive parity $\mathrm{B}_{s}$ mesons from lattice QCD. Phys. Lett. B 750, 17-21 (2015). doi:10.1016/j.physletb.2015.08.038. arXiv:1501.01646

37. C.B. Lang, D. Mohler, S. Prelovsek, $B_{s} \pi^{+}$scattering and search for X(5568) with lattice QCD. Phys. Rev. D 94(074), 509 (2016). doi:10.1103/PhysRevD.94.074509. arXiv:1607.03185

38. L. Leskovec, S. Prelovsek, Scattering phase shifts for two particles of different mass and non-zero total momentum in lattice QCD. Phys. Rev. D85(114), 507 (2012). doi:10.1103/PhysRevD.85.114507. arXiv:1202.2145

39. C. Liu, X. Feng, S. He, Two particle states in a box and the S-matrix in multi-channel scattering. Int. J. Mod. Phys. A 21, 847-850 (2006). doi:10.1142/S0217751X06032150. arXiv:hep-lat/0508022

40. M. Luscher, Volume dependence of the energy spectrum in massive quantum field theories. 2. Scattering States. Commun. Math. Phys. 105, 153-188 (1986). doi:10.1007/BF01211097

41. M. Luscher, Signatures of unstable particles in finite volume. Nucl. Phys. B364, 237-254 (1991a). doi:10.1016/05503213(91)90584-K

42. M. Luscher, Two particle states on a torus and their relation to the scattering matrix. Nucl. Phys. B354, 531-578 (1991b). doi:10.1016/0550-3213(91)90366-6

43. M. Luscher, U. Wolff, How to calculate the elastic scattering matrix in two- dimensional quantum field theories by numerical simulation. Nucl. Phys. B339, 222-252 (1990). doi:10.1016/0550-3213(90)90540-T

44. C. Michael, Adjoint sources in lattice gauge theory. Nucl. Phys. B 259, 58 (1985). doi:10.1016/0550-3213(85)90297-4

45. D. Mohler, C.B. Lang, L. Leskovec, S. Prelovsek, R.M. Woloshyn, $D_{s 0}^{*}(2317)$ meson and $D$-meson-Kaon scattering from lattice QCD. Phys. Rev. Lett. 111(22), 222001 (2013a). doi:10.1103/PhysRevLett.111.222001. arXiv:1308.3175

46. D. Mohler, S. Prelovsek, R.M. Woloshyn, $D \pi$ scattering and $D$ meson resonances from lattice QCD. Phys. Rev. D 87(3), 034501 (2013b). doi:10.1103/PhysRevD.87.034501. arXiv:1208.4059

47. G. Moir, M. Peardon, S.M. Ryan, C.E. Thomas, D.J. Wilson, Coupled-channel $D \pi, D \eta$ and $D_{s} \bar{K}$ scattering from lattice QCD. JHEP 10, 011 (2016). doi:10.1007/JHEP10(2016)011. arXiv:1607.07093

48. J. Nebreda, Strange and non-strange quark mass dependence of elastic light resonances from SU(3) unitarized chiral perturbation theory to one loop. Phys. Rev. D 81, 054035 (2010). doi:10.1103/PhysRevD.81.054035. arXiv:1001.5237

49. S. Neubert, Review on exotic hadrons. In: 12th Conference on Quark Confinement and the Hadron Spectrum (Confinement XII) Thessaloniki, Greece, 29 Aug-3 Sept (2016)

50. M. Nielsen, R.M. Albuquerque, J.M. Dias, K.P. Khemchandani, A. Martinez Torres, F.S. Navarra, C.M. Zanetti, X, Y and Z States. In: 12th Conference on Quark Confinement and the Hadron Spectrum (Confinement XII) Thessaloniki, Greece, 29 August-3 September, 2016, http://inspirehep.net/record/1496993/files/arXiv:1611.03300, arXiv:1611.03300 (2016)

51. S. Ozaki, S. Sasaki, Lúscher's finite size method with twisted boundary conditions: an application to $J / \psi-\phi$ system to search for narrow resonance. Phys. Rev. D87(014), 506 (2013). doi:10.1103/PhysRevD.87.014506. arXiv:1211.5512

52. M. Padmanath, C.B. Lang, S. Prelovsek, X(3872) and Y(4140) using diquark-antidiquark operators with lattice QCD. Phys. Rev. D 92(3), 034501 (2015). doi:10.1103/PhysRevD.92.034501. arXiv:1503.03257

53. C. Patrignani et al., Review of particle physics. Chin. Phys. C40(10), 100001 (2016). doi:10.1088/1674-1137/40/10/100001

54. M. Peardon et al., A novel quark-field creation operator construction for hadronic physics in lattice QCD. Phys. Rev. D 80(054), 506 (2009). doi:10.1103/PhysRevD.80.054506. arXiv:0905.2160

55. J.R. Pelaez, From controversy to precision on the sigma meson: a review on the status of the non-ordinary $f_{0}(500)$ resonance. Phys. Rep. 658, 1 (2016). doi:10.1016/j.physrep.2016.09.001. arXiv:1510.00653

56. C. Pelissier, A. Alexandru, Resonance parameters of the rho-meson from asymmetrical lattices. Phys. Rev. D87(014), 503 (2013). doi:10.1103/PhysRevD.87.014503. arXiv:1211.0092 
57. M.R. Pennington, Impressions of the meson spectrum: hybrids and exotics, present and future. EPJ Web Conf. 113(01), 014 (2016). doi:10.1051/epjconf/201611301014. arXiv:1509.02555

58. K. Polejaeva, A. Rusetsky, Three particles in a finite volume. Eur. Phys. J. A 48, 67 (2012). doi:10.1140/epja/i2012-120678. arXiv:1203.1241

59. S. Prelovsek, L. Leskovec, Evidence for X(3872) from DD* scattering on the lattice. Phys. Rev. Lett. 111(192), 001 (2013a). doi:10.1103/PhysRevLett.111.192001. arXiv:1307.5172

60. S. Prelovsek, L. Leskovec, Search for $Z_{c}^{+}(3900)$ in the $1^{+-}$channel on the lattice. Phys. Lett. B 727, 172-176 (2013). doi:10. 1016/j.physletb.2013.10.009. arXiv:1308.2097

61. S. Prelovsek, C.B. Lang, L. Leskovec, D. Mohler, Study of the $Z_{c}^{+}$channel using lattice QCD. Phys. Rev. D 91(1), 014504 (2015). doi:10.1103/PhysRevD.91.014504. arXiv:1405.7623

62. K. Rummukainen, S.A. Gottlieb, Resonance scattering phase shifts on a nonrest frame lattice. Nucl. Phys. B450, $397-436$ (1995). doi:10.1016/0550-3213(95)00313-H. arXiv:hep-lat/9503028

63. C.E. Thomas. Meson spectroscopy, resonances and scattering on the lattice. In: 12th Conference on Quark Confinement and the Hadron Spectrum (Confinement XII) Thessaloniki, Greece, 29 Aug-3 Sept (2016)

64. C.E. Thomas, R.G. Edwards, J.J. Dudek, Helicity operators for mesons in flight on the lattice. Phys. Rev. D 85(014), 507 (2012). arXiv:1107.1930

65. D.J. Wilson, R.A. Briceno, J.J. Dudek, R.G. Edwards, C.E. Thomas, Coupled $\pi \pi, K \bar{K}$ scattering in $P$-wave and the $\rho$ resonance from lattice QCD. Phys. Rev. D 92(9), 094502 (2015a). doi:10.1103/PhysRevD.92.094502. arXiv:1507.02599

66. D.J. Wilson, J.J. Dudek, R.G. Edwards, C.E. Thomas, Resonances in coupled $\pi K, \eta K$ scattering from lattice QCD. Phys. Rev. D 91(5), 054008 (2015b). doi:10.1103/PhysRevD.91.054008. arXiv:1411.2004 